\title{
Developpemnt Du Capital Humain, Croissance Economique Et Bien-Etre En Côte d'Ivoire
}

\author{
Dr. Seydou Koné \\ Université Alassane Ouattara de Bouaké, Côte d’Ivoire
}

doi: 10.19044/esj.2016.v12n34p251 URL:http://dx.doi.org/10.19044/esj.2016.v12n34p251

\begin{abstract}
This paper assesses human capital development policies and their impact on economic growth and households' well-being in Côte d'Ivoire. A dynamic computable general equilibrium model was used to measure the impact of public spending on education and health policies as predicted by the government and then the effects of a larger increase of those spending on economic growth and household welfare in Côte d'Ivoire. The simulations results show that public spending in education and health has positive impacts on education and health demands, on the improvement of labor's factor quality and on the productive capacities of poor and vulnerable households. The results also show that there is a positive correlation between public expenditures on education and health, economic growth and welfare in Côte d'Ivoire.
\end{abstract}

Keywords: Human capital, CGE model, economic growth, well-being

\section{Résumé}

Ce papier évalue les politiques de développement du capital humain et leur impact sur la croissance économique et le bien-être des ménages en Côte d'Ivoire. Un modèle d'équilibre général calculable dynamique a été utilisé pour mesurer l'impact des politiques de dépenses publiques d'éducation et de santé telles que prévues par le gouvernement et ensuite les effets d'un accroissement plus important de ces dépenses sur la croissance économique et le bien-être des ménages en Côte d’Ivoire. Les résultats des simulations montrent que les dépenses publiques d'éducation et de santé ont des impacts positifs sur la demande d'éducation et de santé, sur l'amélioration de la qualité du facteur travail et les capacités productives des ménages pauvres et vulnérables. Ils révèlent également qu'il y’a une corrélation positive entre les dépenses publiques d'éducation et de santé, la croissance économique et le bien-être en Côte d'ivoire. 
Mots-clés : Capital humain, modèle d’EGC, croissance économique, bienêtre

\section{Introduction}

Le lien entre le développement du capital humain et la croissance économique n'est pas nouveau dans la littérature économique. Les théoriciens de ce concept érigent l'accumulation de la connaissance et de la santé au rang d'investissement au même titre que tous les autres. Pour Becker (1964), le capital humain regroupe l'ensemble des capacités productives dont dispose un individu par accumulation de connaissances générales ou spécifiques, de savoir-faire tout en tenant compte du maintien de son état physique de santé. Lucas (1988), établit un lien entre le niveau du capital humain et la croissance économique. Pour ce dernier, le niveau de la production est intimement lié au stock de capital humain dont dispose l'économie. Quant aux théoriciens de la croissance endogène (Romer, 1988 ; Acemoglu, 1997), ils mettent l'accent sur le niveau de formation, élément moteur de la croissance économique selon eux. La Côte d'Ivoire, pays qui sort d'une crise militaro politique et qui a amorcé une phase de relance de son économie, ambitionne d'être un pays émergent à l'horizon 2020. Pour atteindre cet objectif, les autorités veulent accroitre de façon significative les dépenses sociales en éducation et en santé améliorant ainsi le développement du capital humain et le bien-être social afin d'avoir une croissance économique inclusive.

La longue crise économique, politique et militaire n’a pas épargné le système éducatif et sanitaire de la Côte d'Ivoire qui s'est fortement dégradé. Par conséquent, les offres d'éducation et de santé sont largement inférieures à leurs demandes respectives. En matière d'éducation, l'objectif est d'atteindre à terme $100 \%$ de scolarisation au primaire et au secondaire ${ }^{18}$. Cela passe par la mise en œuvre de plusieurs mesures notamment la construction de nouvelles salles de classes, le recrutement d'enseignants, la gratuité des manuels scolaires au primaire public, etc. Des efforts sont également prévus pour la formation professionnelle et l'enseignement supérieur. Dans le domaine de la santé, d'importants investissements sont prévus ainsi qu'une aide aux populations défavorisées. Il est également prévu la mise en application de la politique de l'Assurance Maladie Universelle ${ }^{19}$ (AMU) à partir de 2017.

\footnotetext{
${ }^{18}$ Programme Présidentiel : http://www.presidence.ci/presentation/10/education, visité le 16 octobre 2016.

${ }^{19}$ Selon cette politique chaque citoyen devra cotiser 1000F CFA par mois et participer à hauteur de 1000F CFA aux frais de consultation afin bénéficier d'une prise en charge complète des soins de santé.
} 
De nombreux auteurs se sont penchés sur la relation entre les dépenses sociales et la croissance économique tant dans les pays développés que dans les pays en développement. Kocoglu et Rodrigo (2009) font une revue de la littérature sur les contributions du secteur de la santé à la croissance économique dans les pays développés. Dans son étude sur un pays comme les Etats-Unis, Aka (2008) a testé les relations de long terme et les effets de causalité entre les dépenses de santé, d'éducation et la croissance économique. Dans le cas spécifique de la Côte d’Ivoire, Keho (2009) a analysé le rôle de la croissance économique et des dépenses sociales sur la dimension sociale du développement durable de ce pays. Coulibaly (2013) a évalué l'impact des dépenses publiques d'éducation sur la croissance économique également en Côte d'Ivoire. On peut donc remarquer qu'aucune de ces études n'aborde l'effet conjoint des dépenses de santé et d'éducation sur la croissance économique et le bien-être des ménages en Côte d'Ivoire et ce en ayant recours à un modèle d'équilibre général calculable. Cette étude se propose de remédier à cette insuffisance afin de proposer aux décideurs des politiques économiques appropriées.

Les problèmes liés aux dépenses sociales de santé et d'éducation sont importantes et d'une grande ampleur avec des besoins et des attentes énormes. L'on a parfois tendance à se demander s'ils peuvent être résolus à court et moyen termes au regard des ressources budgétaires limitées dont disposent nos Etats. Il est cependant impératif d'évaluer les effets des efforts fournis et de mesurer ce qui reste à faire. Dans ce contexte, l'on pourrait s'enquérir des accroissements des dépenses de santé et d'éducation sur la croissance économique en Côte d’Ivoire ? Ces dépenses sont-elles favorables à l'emploi et à l'accroissement des capacités productives des ménages pauvres et vulnérables ? Contribuent-elles à l'amélioration du revenu de ces ménages et au bien-être des ménages en général ?

L'objectif de cette étude est de mesurer les impacts des dépenses publiques d'éducation et de santé sur la croissance économique et le bienêtre des ménages en Côte d'Ivoire. Subsidiairement, l'étude évalue l'impact de ces dépenses sociales sur l'accès aux services sociaux de base d'éducation et de santé, l'emploi et la capacité productive des ménages pauvres et vulnérables.

La suite de l'étude est articulée de la façon suivante. La section 2 est consacrée à la revue de la littérature. La section 3 décrit le cadre méthodologique. La section 4 procède à l'analyse des résultats des simulations. La section 5 conclut l'étude.

\section{Revue de la littérature}

Les études sur le lien entre les dépenses publiques tendant à améliorer le capital humain, la croissance économique et le bien-être des 
ménages, peut se subdiviser en trois groupes. Le premier est relatif à l'impact des dépenses sociales d'éducation et de santé sur la croissance économique et le bien-être des ménages, le second traite de la relation entre les dépenses d'éducation et la croissance et enfin le troisième de l’influence des dépenses de santé sur la croissance et le bien-être des ménages.

Certains auteurs considèrent les dépenses sociales comme les plus importantes des dépenses publiques car ayant un impact en dernier ressort sur le bien-être des populations. Pour Dvandewalle (1999), l'un des objectifs les plus importants des dépenses publiques est d’améliorer le bien-être des populations et en particulier celui des plus pauvres. Pour cet auteur, l'évaluation des dépenses peut se faire en utilisant deux groupes de méthodes. Le premier groupe étudie les incidences des dépenses publiques le second groupe adopte des approches comportementales. Cueta, Kaboso et Suarez-Bererra (2012), dans le cas spécifique de l’économie zambienne, se focalisent sur l'incidence des dépenses sociales traditionnelles que sont l'éducation, la santé, les soutiens aux agriculteurs modestes. Leurs résultats indiquent que les dépenses publiques d’éducation ne bénéficient pas aux pauvres mais sont progressives. Par contre, les dépenses de santé, en plus de ne profiter aux pauvres sont régressives. Keho (2009), dans une étude sur la Côte d'Ivoire, utilise des modèles autorégressifs et des tests de causalité de Granger pour montrer le rôle de la croissance et des dépenses sociales sur la dimension sociale du développement durable. Ses résultats indiquent qu’à long terme il y’a une absence de causalité entre le PIB, les dépenses publiques et les indicateurs d'éducation. L'auteur trouve que la croissance économique est importante pour le développement humain et que le pays devra maintenir son taux à un niveau de même que le niveau des dépenses sociales pour faire face à la demande d’éducation et de santé d'une population croissante.

Quant au second groupe d'études sur la relation entre les dépenses d'éducation et la croissance économique, il montre entre autre qu'un accroissement marginal des dépenses publiques d'éducation est source de croissance durable et de réduction de la pauvreté au Pérou (Gustavo, Castro, Beltran et Cardenas, 2008). Dans le même ordre d’idée, Coulibaly (2013) montre qu'en Côte d'Ivoire l'accroissement des dépenses publiques d'éducation a significativement un impact positif sur la croissance économique. Audet, Makdissi et Wodon (2006) utilisant une méthode à dominance stochastique, arrivent à la même conclusion non sans préciser qu'une réallocation des dépenses publiques d’éducation vers le primaire et le secondaire est source de réduction de la pauvreté en Côte d'Ivoire.

Au-delà des effets macroéconomiques, certains auteurs ont analysé l'impact des dépenses d'éducation sur le bien-être des ménages à faible revenu. Duclos, Kiconco, Levine, Enyimu, Rodriguez et Musisi (2013) dans 
leur étude sur les programmes d'éducation universelle en Ouganda, révèlent que grâce à ces actions, le taux de scolarisation a été progressif. De plus, ces programmes ont été bénéfiques aux pauvres dans le temps. Pour maintenir les enfants à l'école surtout dans les régions rurales, les pouvoirs publics initient avec les organismes internationaux tels que la $\mathrm{FAO}^{20}$ des programmes de cantine scolaire. Diagne, Lô, Sokhna, et Diallo (2013) montrent qu'au Sénégal, la cantine scolaire a un impact positif et significatif sur le score global des élèves du grade 2 (10,56 points). Ce résultat est très important car il montre que sans ces aides alimentaires, de nombreux enfants dans les pays africains issus de familles pauvres ou qui résident en zone rurales seraient exclus du système éducatif. Outre les programmes de cantine scolaire, il existe d'autres programmes tendant à améliorer le taux de scolarisation tel que la réallocation de revenu au niveau familial. Ainsi, Amarante , Arim , De Melo, et Vigorito (2010), montrent qu'en Uruguay, la réforme du programme d'allocation visant à réduire la pauvreté et améliorer le taux de scolarisation des enfants de 0 à 18 ans et particulièrement chez les adolescents permet d'accroitre le taux de scolarisation chez cette catégorie de six à huit points de pourcentage. Les effets bénéfiques des politiques d'éducation ne sont pas perceptibles seulement dans les pays à revenu modeste puisque dans un pays en plein essor économique comme la Chine, Xiaohua, Zhao et Lu (2008) ont montré en étudiant les effets de l'éducation sur les inégalités de gain dans les zones urbaines sur la période 1988-2003 que l'inégalité des gains dans les zones urbaines se faisait au profit des populations les plus éduquées. Ces auteurs concluent à l'importance de l'éducation dans l'accroissement du revenu des ménages.

Le troisième groupe d'étude sur le lien entre les dépenses publiques et le capital humain concerne celles qui ont analysées l'impact des dépenses de santé sur la croissance économique et le bien-être des ménages. O’Donnell, Van Doorslaer, Rannan-Eliya, Somanathan, Adhikari, Harbianto, Garg, Hanvoravongchai, Huq, Karan, Leung, Wan, Pande, Tin, Tisayaticom, Trisnantoro, Zhang et Zhao (2007) montrent que dans la plupart des pays développés d'Aie l'augmentation des dépenses publiques de santé est plutôt favorable aux riches qu'aux pauvres. Pour un autre pays développé comme les Etats-Unis, Aka (2008) a testé les relations de long terme et les effets de causalité entre les dépenses de santé et d'éducation et la croissance économique. Il trouve qu'il y’a une relation de causalité entre les dépenses d'éducation et de santé et la croissance économique dans ce pays mais la relation inverse n'est pas vérifiée et que dans le long terme les performances économiques sont en partie expliquées par l'état antérieur de santé et d'éducation.

${ }^{20}$ Organisation Mondiale pour l'agriculture et l'alimentation. 
Les études sur les dépenses de santé ne concernent pas uniquement que leur impact sur la croissance économique. Elles se sont aussi focalisées sur des aspects tels que l'impact de ces dépenses sur le bien-être de la femme et de l'enfant, la progressivité des services de santé (Doumbouya et Kaba, 2009 ; Plan de Développement sanitaire au Niger 2005-2009, 2015). Les questions de l'efficacité des dépenses de santé pour les pauvres ont aussi été abordées par Kamgnia, Wangun, Tatsinkou et Afor, (2008) pour un pays comme le Cameroun. Les résultats de leur étude indiquent que ces dépenses sont favorables aux pauvres. Le dernier aspect analysé par ces études est relatif au coût de la santé et à son financement. Les dépenses de santé sont chères et constitue une part importante du budget des ménages. Au Cameroun, la propension moyenne de la consommation médicale totale des ménages est très élevée et représente $68 \%$ des dépenses totales des ménages. Cela pose le problème du financement des dépenses de santé surtout chez les ménages pauvres. L'une des solutions proposée est l'assurance santé communautaire dans les régions rurales. Au Nigeria, cette ce mode de financement n'ayant pas connu le succès attendu, a conduit Ataguba, Ichoku et Fonta, (2008) à proposer une solution alternative consistant à pré payer les frais de santé au niveau communautaire.

Cette revue de la littérature montre que la question de l'impact des dépenses publiques d'éducation et de santé sur la croissance et le bien-être des ménages pauvres en Côte d'Ivoire a pour l'essentiel été adressée avec des modèles économétriques. Dans cette étude, nous allons utiliser un modèle d'équilibre général calculable dynamique pour évaluer l'effet conjoint des dépenses publiques d'éducation et de santé sur la croissance économique et le bien-être des populations démunis en Côte d'Ivoire.

\section{Méthodologie}

Cette section présente la matrice de comptabilité sociale utilisée dans cette étude et ensuite quelques parties du modèle d'équilibre général utilisé notamment les fonctions de demande, celles des ménages, la dynamique du modèle et les équations d'équilibres macroéconomiques et la fermeture du modèle.

\section{Matrice de comptabilité sociale}

La matrice de comptabilité sociale (MCS) que nous utilisons dans cette étude est celle de la Côte d'Ivoire pour l'année 2007 que nous avons construite. Les sources de données ayant servi à sa construction sont d'origines diverses et issues de l'institut National de Statistique (INS), de la Direction de la Comptabilité Publique et de l'économie, de la Banque Centrale des Etats de l'Afrique de l'Ouest (BCEAO), de la matrice d'offre et de demande pour la Côte d'ivoire, du tableau des comptes économiques 
intégrés de 2007, du tableau des recettes mensuelles, de la Direction générale des Impôts (DGI), de la balance des paiements et du tableau du PIB et de ses emplois au prix courant. Cette matrice comporte 84 comptes regroupés en six catégories : les facteurs de production, les agents, les branches de production, les produits destinés au marché intérieur ou domestique les exportations les taxes et l'accumulation.

Les facteurs de production sont le travail qualifié, le travail non qualifié le capital publique et le capital privé. Dans la MCS on a neuf catégories de ménage, un compte pour le gouvernement, les entreprises ainsi que le reste du monde, dix-neuf branches de production et de produits. Les produits exportables sont au nombre de seize. Un compte respectivement pour les investissements privés, les investissements publics et la variation des stocks.

Les salariés du public et du privé formel tirent principalement leur revenu de la rémunération du travail qualifié (Tableau $1^{21}$ ) tandis que ceux du privé, qu'ils soient du secteur formel et informel, les agriculteurs, les éleveurs, les pêcheurs et les indépendants ont le travail non qualifié comme source principale de revenu. Quant aux inactifs, les transferts provenant des entreprises constituent l'essentiel de leur revenu. On peut aussi remarquer que les salariés, de toutes origines, ont une part non négligeable de leur revenu qui provient du transfert des entreprises. Les transferts du gouvernement eux, sont réparti de la même manière à tous les ménages.

Tableau 1: Source de revenu des ménages

\begin{tabular}{|c|c|c|c|c|c|c|c|}
\hline & $\mathrm{TNQ}^{22}$ & TQ & KPR & $\begin{array}{c}\text { TR } \\
\text { ETS }\end{array}$ & $\begin{array}{c}\text { TR } \\
\text { GVT }\end{array}$ & $\begin{array}{c}\text { TR } \\
\text { RDM }\end{array}$ & TOTAL \\
\hline Salariés du public & 5.00 & 46.41 & 0.52 & 37.00 & 7.44 & 3.63 & 100.00 \\
\hline $\begin{array}{l}\text { Salariés du privé } \\
\text { formel }\end{array}$ & 12.32 & 57.41 & 0.47 & 20.31 & 7.50 & 1.99 & 100.00 \\
\hline $\begin{array}{c}\text { Salariés du privé } \\
\text { informel }\end{array}$ & 35.26 & 29.09 & 0.21 & 25.46 & 7.48 & 2.50 & 100.00 \\
\hline $\begin{array}{c}\text { Agriculteurs } \\
\text { industriels }\end{array}$ & 65.49 & 13.11 & 0.17 & 12.51 & 7.49 & 1.23 & 100.00 \\
\hline Agriculteurs vivriers & 84.03 & 6.34 & 0.00 & 1.91 & 7.53 & 0.19 & 100.00 \\
\hline Eleveurs & 70.62 & 11.03 & 0.00 & 9.86 & 7.51 & 0.97 & 100.00 \\
\hline Pêcheurs & 68.82 & 23.64 & 0.00 & 0.00 & 7.55 & 0.00 & 100.00 \\
\hline $\begin{array}{l}\text { Indépendants et } \\
\text { employés non agri. }\end{array}$ & 54.26 & 35.09 & 0.00 & 2.82 & 7.55 & 0.28 & 100.00 \\
\hline Inactifs & 1.06 & 1.18 & 0.00 & 82.36 & 7.33 & 8.08 & 100.00 \\
\hline
\end{tabular}

Source : Matrice de Comptabilité Sociale

${ }^{21}$ TNQ : Travail non qualifié, TQ : Travail non qualifié, KPR : Capital privé, KPU : Capital public, TR ETS : Transfert des entreprises, TR GVT: Transfert du gouvernement, TR $\mathrm{RDM}$ : Transfert en provenance du reste du monde.

${ }^{22}$ TNQ : Travail non qualifié, TQ : Travail non qualifié, KPR : Capital privé, KPU : Capital public, TR ETS : Transfert des entreprises, TR GVT : Transfert du gouvernement, TR RDM : Transfert en provenance du reste du monde. 
Si les sources de revenu ci-dessus varient selon la catégorie socioprofessionnelle du ménage, on peut noter que tous consacrent une part importante de ce revenu à la consommation de produits de l'agriculture vivrière, de produits de l'industrie agro-alimentaire, et de produits industriels en général (Tableau 2). Les biens de l'industrie textile et de l'habillement viennent également compléter cette gamme de produits citée. Les services, ceux de transport et de communication en particulier occupent aussi une grande part dans le budget de consommation des ménages.

Tableau 2: Consommation des ménages

\begin{tabular}{cccccccccc}
\hline & $\begin{array}{c}\text { SALPUB } \\
\mathbf{2 3}\end{array}$ & SALPRI & SECIN & AGRIN & AGR & ELE & PEC & IND & INA \\
& & F & D & V & V & H & & C \\
\hline AVI & 12.72 & 15.56 & 17.95 & 21.11 & 20.70 & 19.63 & 20.85 & 15.98 & 15.80 \\
AEI & 1.94 & 2.37 & 2.74 & 3.22 & 3.16 & 3.00 & 3.18 & 2.44 & 2.41 \\
ECH & 1.98 & 2.42 & 2.79 & 3.28 & 3.22 & 3.05 & 3.24 & 2.49 & 2.46 \\
SYL & 1.11 & 0.85 & 2.19 & 0.31 & 0.63 & 0.58 & 0.00 & 7.28 & 1.59 \\
PCH & 1.04 & 1.27 & 1.47 & 1.73 & 1.69 & 1.60 & 1.70 & 1.31 & 1.29 \\
XTR & 0.00 & 0.00 & 0.00 & 0.00 & 0.00 & 0.00 & 0.00 & 0.00 & 0.00 \\
IAA & 13.92 & 17.02 & 19.64 & 23.10 & 22.66 & 21.48 & 22.82 & 17.49 & 17.29 \\
\hline TEX & 2.72 & 2.99 & 2.69 & 2.86 & 2.90 & 3.08 & 3.27 & 2.53 & 2.06 \\
OAI & 17.45 & 18.20 & 17.32 & 15.48 & 17.44 & 19.40 & 20.27 & 15.30 & 17.85 \\
\hline EGE & 1.07 & 1.30 & 1.20 & 0.38 & 0.75 & 0.59 & 0.63 & 1.09 & 1.96 \\
CO & 1.99 & 0.61 & 0.41 & 0.38 & 0.68 & 0.48 & 1.59 & 0.13 & 0.17 \\
N & & & & & & & & & \\
CO & 0.00 & 0.00 & 0.00 & 0.00 & 0.00 & 0.00 & 0.00 & 0.00 & 0.00 \\
M & & & & & & & & & \\
HOT & 2.69 & 2.80 & 2.67 & 2.38 & 2.69 & 2.64 & 3.12 & 2.38 & 2.80 \\
\hline TCO & 16.76 & 17.07 & 13.60 & 6.73 & 10.17 & 10.26 & 7.79 & 13.12 & 15.26 \\
FIN & 2.01 & 2.10 & 2.00 & 1.78 & 2.01 & 1.98 & 2.33 & 1.78 & 2.10 \\
\hline EDU & 0.52 & 0.44 & 0.26 & 0.17 & 0.26 & 0.22 & 0.20 & 0.26 & 0.71 \\
SAN & 0.49 & 0.48 & 0.41 & 0.44 & 0.46 & 0.43 & 0.50 & 0.30 & 0.56 \\
\hline OSV & 8.46 & 8.83 & 8.40 & 7.50 & 8.46 & 9.25 & 4.92 & 7.43 & 8.82 \\
\hline Tota & 100.00 & 100.00 & 100.00 & 100.00 & 100.0 & 100.0 & 100.0 & 100.0 & 100.0 \\
l & & & & & 0 & 0 & 0 & 0 & 0 \\
\hline
\end{tabular}

Source : Matrice de Comptabilité Sociale

${ }^{23}$ Salariés du public (SALPUB), Salariés du privé formel (SALPRIV), Salariés du privé informel (SECINF), Agriculteurs industriels (AGRIND), Agriculteurs vivriers (AGRV), Eleveurs (ELEV), Pêcheurs (PECH), Indépendants (IND), Chômeurs et inactifs (INA).

${ }^{24}$ Agriculture vivrière (AVI) Agriculture industrielle et d'exportation (AEI) élevage et chasse (ECH) Sylviculture et exploitation forestière (SYL), Pêche (PCH), Activités extractives (XTR), Industrie agroalimentaire (IAA), Textile cuire et habillement (TEX), Autres activités industrielles (OAI), Electricité eau et gaz (EGE), Construction (CON), Commerce (COM), hébergement et restauration (HOT), Transport et communication (TCO), Activités financières (FIN), Administration publique (GVT), Education (EDU), Santé et action sociale (SAN), Autres services (OSV). 
Le Tableau 3 ci-dessous indique que sur les 19 branches de production, 5 sont intensives en capital (industrie extractive, industrie agroalimentaire, énergie eau et gaz, commerce, transport et communication). Les 14 autres sont intensives en facteur travail. Toutefois, les branches de l'agriculture vivrière, de l'agriculture industrielle et d'exportation, de l'élevage et de la chasse, de la pêche, et de l'hôtellerie sont plutôt intensives en travail non-qualifié.

Tableau 3: Rémunération des facteurs de production par branche (\%)

\begin{tabular}{cccccc}
\hline & TNQ & TQ & KPR & KPU & TOTAL \\
\hline AVI & 92.81 & 7.19 & 0.00 & 0 & 100 \\
AEI & 76.95 & 15.81 & 7.23 & 0 & 100 \\
ECH & 82.82 & 13.29 & 3.89 & 0 & 100 \\
SYL & 41.56 & 51.27 & 7.17 & 0 & 100 \\
PCH & 69.49 & 24.51 & 6.00 & 0 & 100 \\
XTR & 2.85 & 6.09 & 91.06 & 0 & 100 \\
IAA & 12.13 & 26.18 & 61.70 & 0 & 100 \\
TEX & 48.48 & 11.96 & 39.55 & 0 & 100 \\
OAI & 31.42 & 22.62 & 45.96 & 0 & 100 \\
EGE & 1.50 & 36.20 & 62.30 & 0 & 100 \\
CON & 35.11 & 37.97 & 26.92 & 0 & 100 \\
COM & 32.19 & 10.26 & 57.55 & 0 & 100 \\
HOT & 59.07 & 40.93 & 0.00 & 0 & 100 \\
TCO & 27.24 & 20.87 & 51.89 & 0 & 100 \\
FIN & 14.69 & 85.31 & 0.00 & 0 & 100 \\
APU & 4.74 & 45.24 & 0.00 & 50 & 100 \\
EDU & 1.64 & 98.36 & 0.00 & 0 & 100 \\
SAN & 8.14 & 83.05 & 8.81 & 0 & 100 \\
OSV & 41.33 & 36.66 & 22.01 & 0 & 100 \\
\hline
\end{tabular}

Source : Matrice de Comptabilité Sociale

\section{Le Modèle}

Dans cette étude, le modèle que nous utilisons est une adaptation du modèle d'équilibre général calculable dynamique séquentiel développé par Decaluwé, Lemelin, Maisonnave, et Robichaud (2010). Dans les lignes suivantes, nous présenterons les équations relatives aux ménages, la dynamique du modèle, les équations d'équilibre et la fermeture du modèle.

\section{Les ménages}

Dans notre modèle, les ménages ont pour source de revenu la rémunération du travail (qualifié et non qualifié), du capital ainsi que les transferts reçus des autres agents c'est-à-dire les entreprises, le gouvernement et le reste du monde. Ainsi on a :

$$
R M_{h, t}=R M T_{h, t}+R M C_{h, t}+R M T R_{h, t}
$$

où : 
$R M_{h, t}:$ Revenu total de la catégorie de ménage $\mathrm{h}$

$R M T_{h, t}:$ Revenu du travail de la catégorie de ménage $\mathrm{h}$

$R M C_{h, t}$ : Revenu du capital de la catégorie de ménage $\mathrm{h}$

$R_{M T R_{h, t}}:$ Transfert reçu par le ménage $\mathrm{h}$ des autres agents

Pour chaque catégorie de ménage, le revenu du travail est distribué proportionnellement entre travail qualifié et travail non qualifié. De même, le revenu du capital est distribué aux agents dont les ménages capitalistes. En définitive, les ménages reçoivent une proportion du transfert versé aux agents. On a donc les équations suivantes :

$$
\begin{aligned}
& R M T_{h, t}=\sum_{l} \lambda_{h, l}^{w l}\left(w_{l, t} \sum_{j} D T_{l, j, t}\right) \\
& R M C_{h, t}=\sum_{k} \lambda_{h, k}^{R K}\left(R_{k, j, t} \sum_{j} D C_{k, j, t}\right) \\
& R M T R_{h, t}=\sum_{a g} T R_{h, a g, t}
\end{aligned}
$$

où :

$D T_{l, j, t}$ : Demande de travail de typel par la branche $\mathrm{j}$

$D C_{k, j, t}$ : Demande de capital de type k par la branche $\mathrm{j}$

$R_{k, j, t}$ : Taux de rendement du capital de type k dans l' industrie $\mathrm{j}$

$T R_{h, a g, t}$ : Transfert des agents ag à la catégorie de ménage $\mathrm{h}$

$w_{l, t}$ : Taux de salaire du type de travail 1

$\lambda_{a g, k}^{R K}$ : Part de revenue du type de capital k reçue par l'agent ag

$\lambda_{h, l}^{W L}$ : Part de revenu du travail $l$ reçue par la catégorie de ménage $\mathrm{h}$

On détermine le revenu disponible des ménages en retranchant les impôts sur le revenu prélevé par le gouvernement. Le budget que chaque ménage consacre à la consommation est la différence entre ce revenu disponible et l'épargne des ménages et la somme des transferts aux autres agents non étatiques. Pour terminer, on peut remarquer que l'épargne des ménages est fonction de l'indice des prix à la consommation et du revenu disponible.

$$
\begin{aligned}
& Y D M_{h, t}=Y M_{h, t}-T D M_{h, t}-T R_{g v t, h, t} \\
& C T M_{h, t}=Y D M_{h, t}-S M_{h, t}-\sum_{a g n g} T R_{a g n g, h, t} \\
& S M_{h, t}=P I X C O N_{t}^{\eta} \operatorname{shO} O_{h, t}+\operatorname{sh} 1_{h} Y D M_{h, t} \\
& \text { où }
\end{aligned}
$$


$C T M_{h, t}$ : Budget de Consommation de la catégorie de ménage h

$\mathrm{PIXCON}_{t}^{\eta}$ : Indice des prix à la consommation

$S M_{h, t}$ : Epargne de la catégorie de ménage $\mathrm{h}$

$T D M_{h, t}$ : Impôt sur le revenu de la catégorie de ménage $\mathrm{h}$

$Y D M_{h, t}$ : Revenu disponible de la catégorie de ménage h

$\eta$ : Paramètre d'élasticité prix des transferts indexés

$s h O_{h, t}$ : ordonnée à l'origine (épargne de la catégorie de ménage h)

$s h 1_{h, t}$ : Pente (épargne de la catégorie de ménage h)

agng : Indice des agents non membres du gouvernement

\section{La dynamique du modèle}

Les équations de la dynamique du modèle sont donc les suivantes :

$O T_{l, t+1}=O T_{l, t}\left(1+n_{t}\right)$

$I N D_{k, p u b, t+1}=I N D_{k, p u b, t}\left(1+n_{t}\right)$

$D C_{k, j, t+1}=D C_{k, j, t}\left(1-\delta_{k, j}\right)+I N D_{k, j, t}$

$O T_{l, t}$ : Offre de travail de type là la période $\mathrm{t}$

$I N D_{k, p u b, t}$ : Volume de nouveaux investissements publics de type $\mathrm{k}$ à la période $\mathrm{t}$

$I N D_{k, j, t}$ : Volume de nouveaux investissements de type $\mathrm{k}$ dans la branche $\mathrm{j}$ à la période $\mathrm{t}$

$n_{t}$ : Taux de croissance de la population.

$\delta_{k, j}:$ Taux de dépréciation du capital

On fait l'hypothèse que l'offre de travail, le volume des investissements et la variation des stocks et la population croissent au même rythme. L'accumulation du capital à la période t est égale au capital de la période précédente diminué de la dépréciation de celui-ci auquel s’ajoute le volume des investissements.

\section{Equilibres macroéconomiques et fermeture du modèle}

Nous faisons l'hypothèse que le marché domestique des biens et services est en équilibre c'est-à-dire l'offre globale de biens et services sur ce marché est égale à la demande. Le marché du travail et celui des capitaux sont aussi supposés être en équilibre. Il y’a aussi égalité entre l'épargne et l'investissement. Le solde de la balance courante est fixe de même que le taux de change et les dépenses publiques. L'hypothèse que la Côte d'ivoire 
est un petit pays ne pouvant influencer les prix mondiaux est également retenue.

\section{Scénarios et Analyse des résultats}

Nous avons retenu deux simulations pour évaluer l'impact des dépenses publiques d'éducation et de santé sur la croissance et le bien-être des ménages. Le premier mesure l'impact des politiques de dépenses en éduction et en santé de l'Etat tel que présenté dans le tableau 4 ci-dessous.

Tableau 4 : Plan de simulation scénario 1 (dépense de santé et d'éducation en \% du PIB)

\begin{tabular}{cccccc}
\hline & 2016 & 2017 & 2018 & 2019 & 2020 \\
\hline Dépense d'éducation (\% du PIB) & 4.88 & 4.95 & 5.05 & 5.08 & 5.14 \\
Dépense de santé (\% du PIB) & 5.8 & 5.55 & 5.06 & 5.11 & 8.32 \\
\hline
\end{tabular}

Source : calculs de l'auteur à partir du PND 2016-2020

La revue de littérature a montré que les dépenses de santé et d'éducation ont un impact positif sur la croissance et le bien-être des populations. Dans le second scénario nous voulons mesurer l'effet sur une période de dix années (2016-2025) de l'accroissement des dépenses d'éducation de santé. Nous simulons un accroissement de ces dépenses à un niveau de 7,5\% du PIB pour les dépenses d'éducation et de $10 \%$ pour les dépenses de santé.

Tableau 5 : dépense d'éducation et de santé (\% du PIB)

\begin{tabular}{ccccccccccc}
\hline & 2016 & 2017 & 2018 & 2019 & 2020 & 2021 & 2022 & 2023 & 2024 & 2025 \\
\hline $\begin{array}{c}\text { Dép. } \\
\text { d'éducation }\end{array}$ & 7.5 & 7.5 & 7.5 & 7.5 & 7.5 & 7.5 & 7.5 & 7.5 & 7.5 & 7.5 \\
$\begin{array}{c}\text { Dépense de } \\
\text { santé }\end{array}$ & 10 & 10 & 10 & 10 & 10 & 10 & 10 & 10 & 10 & 10 \\
\hline
\end{tabular}

Source : Estimations de l'auteur

La présentation de ces scénarios de simulation est suivie dans les lignes qui vont suivre par la présentation et l'analyse des résultats de ceux-ci.

\section{Simulation 1}

Dans ce scénario, l'on suppose que les dépenses de santé et d'éducation sont financées par le prélèvement de taxes sur la production ${ }^{25}$.

${ }^{25}$ Les autorités ivoiriennes dans le cadre du PND 2016-2020 tablent sur un accroissement de la production industrielle. Cette hausse si elle est effective sera source de revenu pour l'Etat du fait des taxes sur la production. 
L’augmentation des dépenses publiques de d'éducation et de santé, comme nous l'avions évoqué plus haut a pour objectif d'avoir une croissance inclusive; cela implique tout d'abord l'accroissement du PIB mais également un meilleur accès aux services sociaux de base, pus d'emplois surtout pour les jeunes et l'amélioration de des capacités productives des ménages pauvres et vulnérables. Les résultats de la simulation une doivent se traduire par un accroissement de la demande de santé et d'éducation qui traduiront un meilleurs accès des populations en général les pauvres aux services sociaux se santé et d'éducation. On doit aussi avoir une augmentation de la production dans les branches agricoles surtout celle de l'agriculture vivrière sensée être la branche de productions des produits par les populations pauvres. Ce résultat sera la preuve de l'amélioration de la capacité productive des ménages pauvres et vulnérables. On s'attend également à avoir comme résultat, un accroissement de la demande de travail par les entreprises et l'Etat.

Tableau 6 : Impact macro-économiques (variation en pourcentage par rapport au scénario de référence)

Source : Calculs de l'auteur

\begin{tabular}{ccccccc}
\hline & 2016 & 2017 & 2018 & 2019 & 2020 & Moyenne \\
\hline $\begin{array}{c}\text { Taux de salaire travail } \\
\quad \text { qualifié }\end{array}$ & -3.45 & -4.27 & -3.52 & -3.78 & -4.27 & -3.86 \\
$\begin{array}{c}\text { Taux de salaire travail non } \\
\text { qualifié }\end{array}$ & 4.53 & 5.08 & 4.02 & 4.10 & 5.13 & 4.57 \\
$\quad \begin{array}{c}\text { Demande d'éducation } \\
\quad \text { Demande de santé }\end{array}$ & 20.62 & 21.35 & 20.65 & 20.87 & 21.29 & 20.95 \\
$\begin{array}{c}\text { Production agriculture } \\
\quad \text { vivrière }\end{array}$ & 19.69 & 19.69 & 19.57 & 19.54 & 19.54 & 19.60 \\
$\quad \begin{array}{c}\text { Production agriculture } \\
\quad \text { industrielle }\end{array}$ & -4.16 & 25.97 & 22.51 & 23.24 & 25.79 & 24.13 \\
Produit Intérieur Brut (PIB) & 2.40 & -4.84 & -4.50 & -4.68 & -5.39 & -4.76 \\
\hline
\end{tabular}

On observe une baisse continuelle du taux de salaire du travail qualifié entre 2016 et 2020 ; ce taux est passé en effet de $-3.45 \%$ à $-4.27 \%$. Le taux moyen sur la période s'établissant à $-3.86 \%$. Le taux de salaire du travail non-qualifié en revanche connait une augmentation avec une tendance à la hausse à partir de 2016. La moyenne sur la période étant de $+4.57 \%$. Ce résultat peut s'expliquer par le fait que l'accroissement des dépenses publiques d'éducation et de santé entraine une hausse du nombre de personnes éduquées et de ce fait l'offre de travail qualifié. Cela aura pour effet, toutes choses étant égale par ailleurs, une baisse du taux de travail qualifié. La hausse de l'offre de travail qualifié s'accompagne de la raréfaction de travailleurs non-qualifiés (la dotation totale dans l'économie du facteur travail étant fixe pour une année donnée). La baisse du taux de salaire de travail qualifié devrait pousser les entreprises à demander une plus grande quantité de ce facteur. Le rendement du travail qualifié étant plus 
élevé que celui du travail-non-qualifié, on peut s'attendre à une production plus grande de biens et services et aussi un changement dans la structure de production en faveur de produits et services intensifs en travail qualifié avec une plus grande valeur ajoutée.

Quant aux demandes d'éducation et de santé, elles ont augmentées de façon notable sur la période avec un taux moyen de 20.95\% par rapport au scénario de référence pour la demande d’éducation et $19.60 \%$ pour la demande de santé. Ce résultat peut s’interpréter comme la conséquence d'une hausse de l'offre d'éducation et de santé impulsée par les pouvoirs publics à travers la construction de salles de classes, de structures de santé, le recrutement d'enseignants et de personnels de santé. Il est en outre conforme aux attentes du gouvernement qui avait pour objectif d'accroître la demande de santé et d'éducation et permettre de ce fait une croissance plus inclusive.

On constate aussi que la production agricole de produits vivriers augmente considérablement sur la période (24.13\%) tandis que celle des produits issus de l'agriculture industriels et d'exportation baisse de $4.76 \%$ en moyenne sur la période. On peut penser que les capacités productives des acteurs de ce secteur qui sont pour la grande majorité des ménages pauvres et vulnérables s'est améliorée. On note toutefois que la forte hausse de la production vivrière s'est faite au détriment de celle de l'agriculture industrielle et d'exportation qui a légèrement baissée.

Quant au PIB, il enregistre une hausse par rapport à celui du scénario de référence qui a tendance à décroître dans le temps. Sur la période, le taux de croissance moyen par rapport au scénario de référence est de 3.60\%. Si dans l'ensemble les variables macroéconomiques sont positivement affectées par la hausse des dépenses sociales d'éducation et de santé, qu'en est-il de son impact sur les ménages ?

Tableau 7 : Part du budget consacrée à la consommation (variation en pourcentage par rapport au scénario de référence).

\begin{tabular}{|c|c|c|c|c|c|c|c|c|c|}
\hline & $\begin{array}{c}\text { SALPU } \\
\text { B } \\
\end{array}$ & $\begin{array}{c}\text { SALPRI } \\
\mathrm{V}\end{array}$ & $\begin{array}{c}\text { SECIN } \\
\text { F }\end{array}$ & $\begin{array}{c}\text { AGRIN } \\
\text { D } \\
\end{array}$ & $\begin{array}{c}\text { AGR } \\
\text { V }\end{array}$ & $\begin{array}{c}\text { ELE } \\
\mathrm{V}\end{array}$ & $\begin{array}{c}\text { PEC } \\
\mathrm{H}\end{array}$ & $\begin{array}{l}\text { IN } \\
\text { D }\end{array}$ & $\begin{array}{c}\text { INA } \\
\text { C }\end{array}$ \\
\hline 2016 & 5.40 & 3.53 & 2.04 & 1.35 & 3.62 & 1.97 & 2.57 & $\begin{array}{c}1.2 \\
0\end{array}$ & 9.10 \\
\hline 2017 & 6.17 & 4.15 & 2.36 & 1.48 & 4.03 & 2.18 & 2.78 & $\begin{array}{c}1.2 \\
1\end{array}$ & $\begin{array}{c}10.0 \\
6\end{array}$ \\
\hline 2018 & 5.63 & 3.73 & 2.36 & 0.94 & 3.18 & 1.55 & 2.20 & $\begin{array}{c}0.8 \\
9\end{array}$ & 9.48 \\
\hline 2019 & 5.94 & 3.97 & 2.54 & 0.90 & 3.22 & 1.53 & 2.20 & $\begin{array}{c}0.8 \\
3\end{array}$ & 9.93 \\
\hline 2020 & 6.73 & 4.45 & 2.72 & 1.34 & 4.07 & 2.08 & 2.84 & $\begin{array}{c}1.2 \\
1\end{array}$ & $\begin{array}{c}11.3 \\
0\end{array}$ \\
\hline $\begin{array}{c}\text { Moyen } \\
\text { ne }\end{array}$ & 5,97 & 3.97 & 2.40 & 1.20 & 3.62 & 1.86 & 2.52 & $\begin{array}{c}1.0 \\
7\end{array}$ & 9.97 \\
\hline
\end{tabular}

Source : Calculs de l'auteur 
Les résultats de nos simulations indiquent que toutes les catégories de ménage enregistrent une hausse de la part de leur budget qu'ils consacrent à la consommation de biens et services (Tableau 7). Deux groupes semblent cependant se distinguer. Il y'a d'une part, les salariés du public, ceux du privé formel, les agriculteurs de vivrier, et les inactifs qui enregistrent une forte hausse (au-delà de 3\% entre 2016 et 2020) et d'autre part les salariés du privé informel, les agriculteurs industriel et d'exportation, les éleveurs et les indépendants dont l'augmentation du budget consacré à la consommation est sur la période inférieure en moyenne à $3 \%$.

Cet accroissement de la possibilité de consommation des ménages dans l'ensemble peut s'expliquer par le fait qu'ils consacrent désormais une part plus faible de leur revenu à financer des dépenses d'éducation et de santé car l'Etat a accru ses dépenses dans ces secteurs ; ce qui profite aux ménages. L'accroissement de la consommation des ménages sera bénéfique pour la production nationale à condition que celle-ci s'adapte pour saisir l'opportunité qui est offerte. Cette augmentation de la production nationale aura en définitive, un impact positif sur la croissance économique comme nous le soulignions un peu plus haut dans le paragraphe précédent.

Pour l'ensemble des ménages, l'augmentation des dépenses publiques de santé et d'éducation en termes de bien-être est bénéfique (Tableau 8).

Tableau 8 : Variation équivalente du revenu ${ }^{26}$

\begin{tabular}{cccccccccc}
\hline & $\begin{array}{c}\text { SALPU } \\
\text { B }\end{array}$ & $\begin{array}{c}\text { SALPRI } \\
\text { V }\end{array}$ & $\begin{array}{c}\text { SECIN } \\
\text { F }\end{array}$ & $\begin{array}{c}\text { AGRIN } \\
\text { D }\end{array}$ & $\begin{array}{c}\text { AGR } \\
\text { V }\end{array}$ & $\begin{array}{c}\text { ELE } \\
\text { V }\end{array}$ & $\begin{array}{c}\text { PEC } \\
\text { H }\end{array}$ & $\begin{array}{c}\text { IN } \\
\text { D }\end{array}$ & $\begin{array}{c}\text { INA } \\
\text { C }\end{array}$ \\
\hline $\begin{array}{c}201 \\
6\end{array}$ & 16 & 18 & 16 & 14 & 20 & 15 & 29 & 21 & 13 \\
201 & & & & & & & & & \\
7 & 16 & 18 & 16 & 15 & 17 & 16 & 30 & 21 & 14 \\
201 & & & & & & & & & \\
8 & 6 & 7 & 3 & 16 & -16 & 19 & 54 & 4 & 4 \\
201 & & & & & & & & & \\
9 & 8 & 8 & 8 & 11 & 1 & 11 & 17 & 11 & 7 \\
202 & & & & & & & & & \\
0 & 16 & 16 & 13 & 0.49 & 2 & 3 & 24 & 3 & 17 \\
\hline
\end{tabular}

Source : Calculs de l'auteur

${ }^{26}$ La variation équivalente du revenu VE est ce qu'il faut soustraire (ajouter) du revenu initial pour qu'au système de prix initial, l'agent ait le même niveau de bien être qu'au système de prix final avec le revenu initial. Une variation équivalente positive correspond à une amélioration et une variation équivalente négative une détérioration.

$V E=Y D M_{1}\left[\prod_{i}\left(\frac{P_{i}^{0}}{P_{i}^{1}}\right)\right]-Y D M_{0}$ où YDM représente le revenu disponible des ménages avant et après le choc, $P_{i}$ les prix des biens composites et $\beta_{i}$ la part relative de la consommation du bien i dans la consommation totale des ménages. 
La simulation 1 concernant les prévisions du gouvernement ivoirien en matière de dépenses publiques d'éducation et de santé a montré que celles-ci auront un effet positif sur l'offre des services sociaux de base que sont l'éducation et la santé, l'emploi, l'amélioration des capacités productives des ménages pauvres et vulnérables. Les dépenses de santé sont cependant en deçà des engagements pris par les pays africains à Abuja en 2001 de consacrer 15\% des budgets nationaux à la santé. De même, les dépenses d'éducation en pourcentage du PIB sont inférieures à celles pratiquées dans les pays africains les plus performants dans le domaine tels que le Sénégal, l'Afrique du sud ou le Botswana qui ont des dépenses publiques d'éducation qui avoisinent $10 \%{ }^{27}$ de leurs PIB.

On propose donc d'analyser les effets de l'accroissement des dépenses de santé et d'éducation tel que proposées dans la simulation 2.

\section{Simulation 2}

Tableau 9 : Impact macro-économiques (variation en pourcentage par rapport au scénario de référence)

\begin{tabular}{llllllllllll}
\hline & 2016 & 2017 & 2018 & 2019 & 2020 & 2021 & 2022 & 2023 & 2024 & 2025 & $\begin{array}{l}\text { Moyenn } \\
\mathrm{e}\end{array}$ \\
\hline wq $^{28}$ & 0.76 & 0.50 & 0.24 & -0.02 & -0.28 & -0.54 & -0.80 & -1.06 & -1.32 & -1.58 & -0.41 \\
wnq & 4.09 & 3.95 & 3.81 & 3.69 & 3.57 & 3.46 & 3.35 & 3.25 & 3.16 & 3.07 & 3.54 \\
DD_ED & 25.3 & 25.6 & 25.8 & 26.1 & 26.4 & 26.6 & 26.9 & 27.2 & 27.4 & 27.7 & \\
U & 4 & & 3 & & & 7 & 4 & 1 & 8 & 6 & 26.54 \\
DD_SA & & & & & & & & & & & \\
N & 29.9 & 29.8 & 29.7 & 29.7 & 29.6 & 29.6 & 29.6 & 29.5 & 29.5 & 29.5 & 29.6 \\
XST- & 23.7 & 24.0 & 24.3 & 24.7 & 25.0 & 25.4 & 25.8 & 26.2 & 26.6 & 27.0 & \\
AVI & 7 & 6 & 7 & 1 & 6 & 3 & 2 & 3 & 5 & 9 & 25.31 \\
XST- & & & & & & & & & & & \\
AIE & -3.67 & -3.87 & -4.08 & -4.27 & -4.47 & -4.66 & -4.84 & -5.02 & -5.20 & -5.38 & -4.54 \\
PIB & & & & & & & & & & & \\
& 4.42 & 4.22 & 4.02 & 3.84 & 3.66 & 3.49 & 3.32 & 3.15 & 2.99 & 2.84 & 3.60 \\
\hline
\end{tabular}

Source : Calculs de l'auteur

Les résultats de l'accroissement des dépenses publiques d'éducation et de santé à un niveau plus important que celui prévu par les autorités ivoiriennes sont différents de ceux obtenus dans la simulation précédente. On a comme dans la première simulation, une baisse du taux de salaire du travail qualifié mais seulement trois ans après le choc avec une ampleur beaucoup

\footnotetext{
${ }^{27}$ UNESCO : rapport sur le financement de l'éducation en Afrique subsaharienne Relever les défis de l'expansion, de l'équité et de la qualité (2011), p.30.

${ }^{28} \mathrm{wq}$ : taux de salaire du travail qualifié, wnq : taux de salaire du travail non-qualifié, DD_EDU : demande d'éducation, DD_SAN : demande de santé, XST-AVI : production agriculture vivrière, XST-AIE : production agriculture industrielle et d'exportation, PIB : Produit Intérieur Brut.
} 
plus faible et en moyenne est de seulement -0,41\% en moyenne par rapport au scénario de référence sur la période 2016-2025. Par contre, le taux de salaire du travail non-qualifié est presque identique à celui de la simulation précédente.

L'accroissement des demandes de santé et d'éducation sont plus importantes (26.54\% et $29.6 \%$ respectivement). Il en est de même pour les productions vivrières et industrielles d'exportation légèrement plus élevées en moyenne dans cette simulation (25.31\% contre $24.13 \%)$ et (-4.54 contre 4.76\%). On observe également un taux de croissance du PIB plus important dont la moyenne est de $3.60 \%$ contre $2.23 \%$ dans la simulation 1 . On peut donc dire qu'il y’a une corrélation positive entre les dépenses publiques d'éducation et de santé et la croissance économique. La section suivante sera consacrée à l'analyse comparée de la part du budget consacrée à la consommation de la simulation 2 par rapport à celle de la simulation 1 .

Tableau 10 : Part du budget consacrée à la consommation

\begin{tabular}{cccccccccc}
\hline & SALPUB & SALPRIV & SECINF & AGRIND & AGRV & ELEV & PECH & IND & INAC \\
\hline 2016 & 5.04 & 3.93 & 2.69 & 1.76 & 3.55 & 2.24 & 3.27 & 2.47 & 8 \\
2017 & 5.41 & 4.23 & 2.98 & 1.56 & 3.41 & 2.05 & 3.11 & 2.29 & 8.67 \\
2018 & 5.77 & 4.52 & 3.25 & 1.36 & 3.28 & 1.87 & 2.96 & 2.12 & 9.19 \\
2019 & 6.12 & 4.81 & 3.53 & 1.17 & 3.15 & 1.7 & 2.82 & 1.95 & 9.71 \\
2020 & 6.48 & 5.09 & 3.8 & 0.99 & 3.03 & 1.54 & 2.69 & 1.78 & 10.22 \\
2021 & 6.83 & 5.38 & 3.06 & 0.81 & 2.91 & 1.38 & 2.56 & 1.62 & 10.73 \\
2022 & 7.18 & 5.66 & 3.32 & 0.64 & 2.81 & 1.22 & 2.43 & 1.47 & 11.23 \\
2023 & 7.52 & 5.94 & 3.58 & 0.47 & 2.7 & 1.07 & 2.31 & 1.31 & 11.73 \\
2024 & 7.87 & 5.22 & 3.84 & 0.31 & 2.6 & 0.92 & 2.19 & 1.16 & 12.22 \\
2025 & 8.21 & 6.5 & 4.09 & 0.15 & 2.51 & 0.78 & 2.08 & 1.01 & 12.72 \\
Moyenne & 6.64 & 5.13 & 3.41 & 0.92 & 3.00 & 1.48 & 2.64 & 1.72 & 10.44 \\
\hline
\end{tabular}

Source : Calculs de l'auteur

La part du budget consacrée à la consommation, tout comme dans la simulation passée augmente pour tous les ménages comme le montre le tableau 10. Une comparaison avec les résultats de la simulation 1 (Tableau 7) permet de faire les remarques suivantes :

- $\quad$ En moyenne, la part du budget consacrée à la consommation dans cette simulation est légèrement plus élevée chez la plupart des ménages à l'exception des éleveurs et les agriculteurs de vivriers. Chez les éleveurs, l'effet du choc est plus important au début et à tendance à diminuer dans le temps ;

- $\quad$ Les salariés du privé informels dans la simulation précédente n’était pas une catégorie de ménage ayant bénéficiée de forte progression du budget consacré à la consommation contrairement à leur situation après cette simulation. De même, les pêcheurs et les indépendants, relativement à la première simulation, connaissent une hausse plus importante de la part du budget qu'ils consacrent à la consommation. 
On note que dans l'ensemble, une augmentation plus importante des dépenses publiques d'éducation et de santé permet aux ménages une consommation plus importante; ce qui serait plus favorable à la consommation intérieure et donc à la production nationale et à une croissance endogène. L'effet de cette seconde simulation sur le bien-être des ménages est présenté dans le tableau suivant.

Tableau 11 : Variation équivalente du revenu

\begin{tabular}{cccccccccc}
\hline & SALPUB & SALPRIV & SECINF & AGRIND & AGRV & ELEV & PECH & IND & INAC \\
\hline 2016 & 11 & 12 & 13 & 23 & 22 & 29 & 12 & 41 & 8 \\
2017 & 10 & 12 & 13 & 21 & 22 & 27 & 12 & 41 & 8 \\
2018 & 10 & 12 & 12 & 18 & -14 & 22 & 14 & 30 & 8 \\
2019 & 9 & 11 & 11 & 16 & 15 & 19 & 17 & 24 & 8 \\
2020 & 9 & 10 & 10 & 14 & 55 & 16 & 21 & 20 & 8 \\
2021 & 9 & 10 & 10 & 13 & 37 & 14 & 27 & 18 & 7 \\
2022 & 8 & 9 & 9 & 12 & 28 & 13 & 16 & 16 & 7 \\
2023 & 8 & 9 & 9 & 11 & 22 & 12 & 10 & 14 & 7 \\
2024 & 8 & 9 & 8 & 10 & 19 & 11 & 9 & 13 & 7 \\
2025 & 8 & 8 & 8 & 10 & 17 & 10 & 7 & 12 & 7 \\
\hline
\end{tabular}

Source : Calculs de l'auteur

On observe ici tout comme dans la première simulation que l'effet de dépenses publiques plus importantes d'éducation et de santé est positif et plus grand pour toutes les catégories de ménage.

\section{Conclusion}

Cette étude a dans un premier temps analysé les effets des politiques de dépenses publiques en éducation et en santé en Côte d'Ivoire et dans un second temps mesuré l'impact de dépenses plus importantes que celles prévues par le gouvernement ivoirien. Pour ce faire, un modèle d'équilibre général calculable dynamique est utilisé pour capter les implications des politiques projetées sur la croissance économique mais également sur l'accès des populations aux services d'éducation et de santé, sur l'emploi et la capacité productive des ménages pauvres et vulnérables.

Les résultats des simulations ont montré que les politiques gouvernementales tels que prévues par le Plan National de Développement PND 2016-2020 auront un impact positif sur la demande d'éducation et de santé ce qui traduirait leur efficacité quand à permettre aux populations d'avoir accès aux services sociaux d'éducation et de santé de base. Ces politiques permettent également d'avoir plus de facteur travail qualifié avec un salaire plus faible ce qui devrait permettre aux entreprises d'avoir accès facilement à ce facteur de production à un coût moindre. Un troisième résultat important est l'amélioration des capacités productives des ménages 
pauvres et vulnérables et au-delà le bien-être de toutes les catégories de ménage. En fin de compte, la politique gouvernementale aura un impact positif sur la croissance économique. On a pu également montrer que des dépenses publiques plus importantes d'éducation et de santé auront un effet encore plus important. De ce fait, l'on invite les autorités à continuer dans leur effort d'amélioration du développement du capital humain et du bienêtre social des populations.

Les résultats ont montrés que les taux de salaire du travail qualifié du fait de l'accroissement des dépenses publiques d'éducation baissent quel que soit le scénario. Cela devrait donc naturellement conduire à une hausse de la demande de travail qualifié de la part des entreprises et même de l'administration public. Cependant, on note un fort taux de chômage des diplômés de l'enseignement supérieur. En effet, nombreux sont ces diplômés qui n'arrivent pas à trouver un emploi. Il serait important pour des recherches futures d'avoir un modèle qui mette l'accent sur le marché du travail.

\section{References:}

1. Acemoglu D. (1997), “Training and Innovation in an Imperfect Labour Marketet”, Review of Economic Studies, n64, pp. 445-464.

2.Aka B.F. (2008), "Health, Education and Economic Growth: Testing For Long-Run Relationships and causal links in the United States", Applied econometrics and International Development, vol-8-2.

3. Alzua M.L., Gasparini L. et Haimovich, F. (2011), "Educational Reform and Labor Market Outcomes: the Case of Argentina's Ley Federal de Educacion", Poverty and Economic Policy Network Working paper, $\mathrm{n}^{\circ} 2011-21$.

4. Amarante V., Arim R., De Melo G. et Vigorito, A. (2009), "Family Allowances and Child School Attendance: an ex-ante Evaluation of Alternative Schemes in Uruguay", Poverty and Economic Policy Network Working paper, $\mathrm{n}^{\circ} 2010-07$.

5. Ataguba J. E., Ichoku H. E. et Fonta, W. (2008), "Estimating the willingness to pay for community healthcare insurance in rural Nigeria”, Poverty and Economic Policy Network Working paper, $\mathrm{n}^{\circ} 2008-10$.

6. Audet M., Makdissi P. et Wodon, Q. (2006), “Analyse de l'incidence des dépenses publiques en éducation en Côte d'ivoire : une approche par dominance stochastique”, Cahier de Recherche CIPREE, n0643.

7. Balma L., Ilboudo W. F. A., Attara A., kaboré R., Zerbo K. et Kaboré, J. S. (2010), "Dépenses publiques d'éducation et pauvreté au Burkina 
Faso : une approche en Equilibre Général Calculable”, Poverty and Economic Policy Network Working paper, n²011-04.

8. Becker G.S. (1064), "Human Capital, A Theoretical and Empirical Analysis”, Columbia University Press for the National Bureau of Economic Research, New York.

9. Coulibaly M. (2013), "Impact des dépenses publiques d’éducation sur la croissance économique en côte d'ivoire”, European Scientific Journal, vol.9, No.25, p. 443-464.

10. Cuesta J., Kabaso P. et Suarez-Becerra, P. (2012), "How pro-poor and progressive is social spending in Zambia?”, World bank , Policy Research Working Paper, $\mathrm{n}^{\circ}$ WPS6052.

11. Dia B. K., Wangun S. L., Tatsinkou C. et Afnor, J. (2008), "Bénéfices acquis et ciblage des pauvres dans les dépenses publiques de santé et d'éducation au Cameroun”, Poverty and Economic Policy Network Working paper, $\mathrm{n}^{\circ} 2008-08$.

12. Diagne A., Lô M. M., Sokhna O. et Diallo, F. (2013), “Evaluation de l'impact des programmes de cantines scolaires sur l'efficacité interne des écoles, les acquisitions cognitives et les capacités d’apprentissage des élèves dans les écoles primaires rurales au Sénégal”, Poverty and Economic Policy Network Working paper, n²013-14.

13. Doumbouya S. F., Kaba A., Keita M., Bah O., Doumbouya M. L. et Camara, K. (2009), "Services sociaux d'éducation et de santé en Guinée: Effets redistributifs de la politique gouvernementale”, Poverty and Economic Policy Network Working paper, n²009-02.

14. Duclos J. Y., Kiconco A., Levine S., Enyimu J., Rodriguez A. W. et Musisi, A. (2013), "Universal Primary Education in Uganda : Equity in opportunities and human capital investment”, Poverty and Economic Policy Network Working paper, n²013-17.

15. Herrera R. (1998), “Dépenses publiques d’éducation et capital humain dans un modèle convexe de croissance endogène”, Revue économique, volume 49, n³, pp. 831-544.

16. Keho Y. (2009), "La dimension sociale du développement durable en Côte d'Ivoire : le rôle de la croissance et des dépenses sociales”, Politique économique et développement, $\mathrm{n}^{\circ}$ 01/2008, pp. 1-23.

17. Kocoglu Y. et Rodrigo, A. D. (2009), "Santé et croissance économique de long terme dans les pays développés : une synthèse des résultats empiriques”, Economie publique, n41-72.

18. Lucas R. E. (1988), “On the Mechanics of Economic Development”, Journal of Monetary Economics, Vol. 21, pp. 3-32.

19. Mallaye D., Van-Guer K. B., komga B. F., Yogo V. T. et Gosngor, E. T. (2015), "Education Language Choice and youth entrepreneurship 
in Chad”, Poverty and Economic Policy Network Working paper, $\mathrm{n}^{\circ} 2015-02$.

20. Moctar S., Salissou M. S., Djibo B. B., Djibo S., N’diaye M. et Boukar, A. M. (2014), “Analyse de l'évolution de l'état sanitaire et du bien-être de l'enfant et de la femme au Niger avec le Plan de developpement Sanitaire (PDS 2005-2009)", Poverty and Economic Policy Network Working paper, $\mathrm{n}^{\circ} 2014-07$.

21. O'Donnell O., Van Doorslaer E., Rannan-Eliya R. P., Somanathan A., Adhikari S. R., Harbianto D., Garg C. C., Hanvoravongchai P., Huq M., Karan A., Leung G. M., Wan Ng C., Pande B. R., Tin K., Tisayaticom K., Trisnantoro L., Zhang Y. et Zhao, Y. (2007), "The Incidence of Public Spending on Healthcare: Comparative Evidence from Asia”, World Bank Economic Revue, n² 21, pp. 93-123.

22. Owundi J.P. (2013), "Poids des Dépenses de Santé sur le Revenu des Ménages au Cameroun”, XXVIIe Congrès international de la population (UIESP) Busan Corea, août (http://iussp.org/fr/event/17/programme/paper/5595).

23. Robichaud V., Tiberti L. et Maisonnave, H. (2011), "Impact of increased public education spending on growth and poverty in Uganda: An integrated micro-macro approach", Poverty and Economic Policy Network Working paper, n²014-01.

24. Romer P. (1986), "Increasing Returns and Long Run Growth", Journal of Political Economy, $n^{\circ}$ 94, pp. 1002-1037.

25. Sahay N., Cockburn J. et Pathak, M. (2013), "Modeling Health in a CGE Framework: A Case Study of India", Poverty and Economic Policy Network Working paper, n²013-07.

26. Van de Walle D. P. (1996), "Assessing the Welfare Impacts of Public Spending”, World Bank Policy Research Working Paper, $\mathrm{n}^{\circ} 1670$.

27. Xiaohua Li X., Zhao Y. et Lu, L. (2008), "Effets de l'éducation sur les inégalités de gain dans les zones urbaines en Chine : 1988 2003”, Poverty and Economic Policy Network Working paper, $\mathrm{n}^{\circ} 2008-09$.

28. Yamuda G., Castro J.F., Beltran A. et Cardenas, M.A. (2008), "Education Attainment, Growth and Poverty Reduction within the MDG Framework: Simulation and Costing for the Peruvian Case", Poverty and Economic Policy Network Working paper, n²008-05. 